\title{
The Phase-Induced Amplitude Coronagraph (PIAA)
}

\section{Olivier Guyon ${ }^{1}$, Eugene A. Pluzhnik ${ }^{1}$, Stephen T. Ridgway ${ }^{2}$, Robert A. Woodruff ${ }^{3}$, Celia Blain ${ }^{1}$, Frantz Martinache ${ }^{4}$, and Raphael Galicher ${ }^{5}$}

\author{
${ }^{1}$ Subaru Telescope, National Astronomical Observatory of Japan, 659 N. A'ohoku Pl., Hilo, HI \\ 96720, USA \\ email: guyon@naoj.org \\ ${ }^{2}$ National Optical Astronomical Observatories,USA \\ ${ }^{3}$ Lockheed Martin Space Corporation, USA \\ ${ }^{4}$ Cornell University, USA \\ ${ }^{5}$ Ecole Normale Superieure, Paris, France
}

\begin{abstract}
The Phase-Induced Amplitude Coronagraph (PIAAC) uses a lossless beam apodization, performed by aspheric mirrors, to produce a high contrast PSF. Thanks to the lossless apodization, this concept offers a unique combination of high theoretical throughput $(\approx 100 \%)$, high angular resolution $(\lambda / d)$, small inner working angle $(\approx 1.5 \lambda / d)$, low chromaticity and low sensitivity to pointing errors or angular star diameter. Together, these characteristics make the PRC an ideal choice for direct imaging of extrasolar terrestrial planets (ETPs) from space. We show that a visible telescope smaller than $4 \mathrm{~m}$ would then achieve the goals of the TPF mission, while other coronagraphs considered for TPF require telescope diameters typically 2 to 3 times larger. On a large size $(8 \mathrm{~m})$ space telescope, ETPs can be searched for around a significantly larger sample of stars, thus enabling a much higher scientific return.
\end{abstract}

Keywords. techniques: high angular resolution, planetary systems.

\section{What is the PIAAC?}

\subsection{Lossless apodization by pupil remapping}

The most convenient way of obtaining a high contrast PSF is to amplitude-apodize the telescope pupil. This is usually done by selective absorption: light is removed mostly from the outer edges of the pupil (see for example Kasdin et al. 2003). Unfortunately, to deliver a $10^{10}$ PSF contrast with this technique, at least $80 \%$ of the light needs to be removed. Since this light is removed from the edges of the pupil, the angular resolution of the telescope is also seriously affected: the Inner Working Angle (IWA) is 3 to $4 \lambda / d$.

An alternative to apodization by selective absorption is apodization by pupil remapping (also referred to as Phase-Induced Amplitude Apodization, PIAA): the geometry of the pupil is "distorded" to obtain the desired amplitude distribution (Guyon 2003, Traub \& Vanderbei 2003, Galicher et al. 2005). To obtain a gaussian-like pupil amplitude profile, the central part of the pupil is to be geometrically shrunk (this increases the surface brightness in the pupil) while the edges of the pupil are streched (decreases the surface brightness).

This operation can be performed by 2 aspheric mirrors which can be placed in a collimated relay beam, as illustrated in figure 1 . The combination of the 2 mirrors is required to maintain a flat wavefront for an on-axis source. 


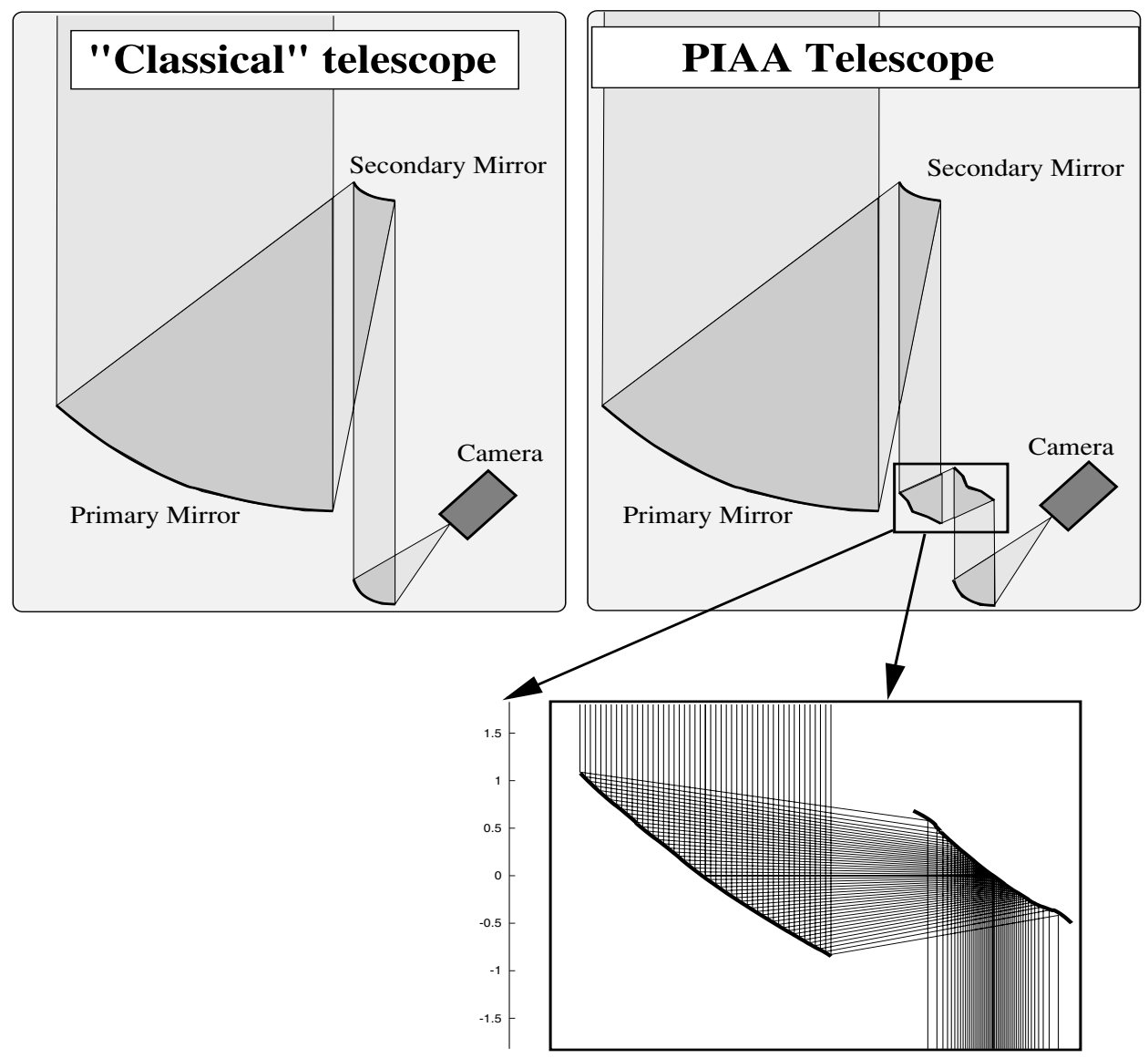

Figure 1. Illustration of the PIAA technique (bottom): a collimated unapodized beam can be amplitude-apodized without light loss thanks to 2 aspheric mirrors. The system preserves the flatness of the wavefront of an on-axis source.

\subsection{The PIAAC design}

The pupil remapping technique described previously is at the heart of the PIAAC design. It is used to produce a gaussian-like (more exactly a prolate spheroidal) output amplitude distribution in the pupil plane. Since the PIAA unit maintains the wavefront flatness of a on-axis point source, the corresponding PSF offers high contrast: most of the light is concentrated in a narrow diffraction core, and the PSF wings can be made fainter than $10^{-10}$ everywhere else.

Unfortunately, the pupil geometric distortion introduced by the PIAA unit is the source of large off-axis PSF aberrations. While the central PSF is sharp and high-contrasted, off-axis sources have PSFs with large coma-like wings. The solution to this problem is to restore the original pupil geometry with a "inverse" PIAA unit after having removed the on-axis light with a focal plane stop. This concept is described in more detail by Guyon (2003), Vanderbei \& Traub (2005) and Guyon et al. (2005).

The light of an on-axis source will be concetrated onto the focal stop by the first PIAA unit. The light of an off-axis source will miss the focal stop, and the second inverse PIAA unit will cancel the effect of the first PIAA unit, therefore maintaining a 

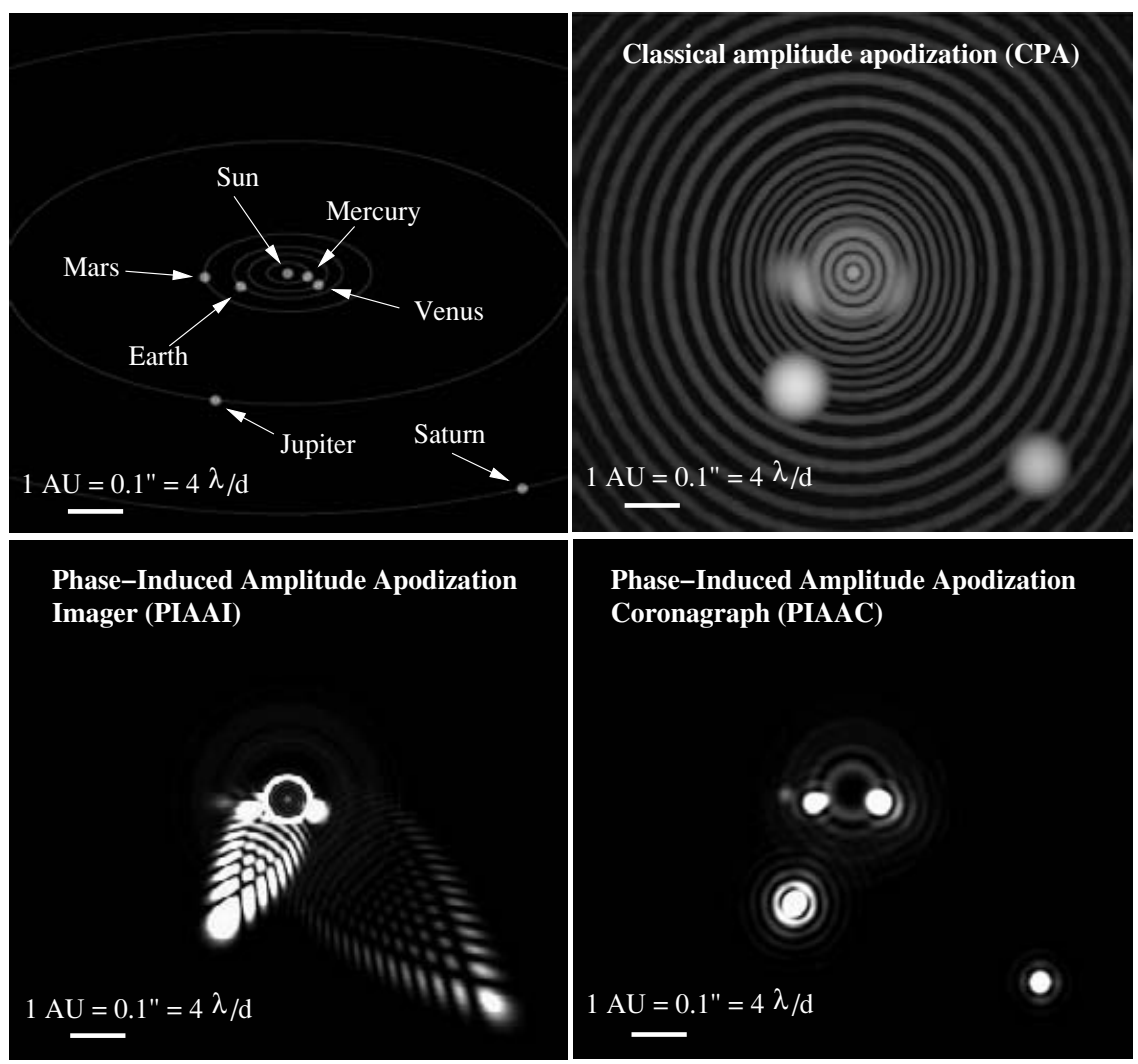

Figure 2. Simulated $0.5 \mu \mathrm{m}$ images of the solar system at a distance of $10 \mathrm{pc}$ with a $4 \mathrm{~m}$ diameter telescope. The same apodization profile was produced by classical apodization (upper right) and PIAA (lower left). In the PIAAC (lower right) the original pupil geometry was restored before imaging. For the CPA and PIAA, an focal plane occulting mask was used to block the starlight, and the images shown are acquired in the reimaged focal plane. In all 3 images, the angular size of the star was taken into account and the faint concentric rings centered on the optical axis are residual starlight.

sharp unaberrated PSF across the field of view. Since this design incorporates a focal plane coronagraphic stop, we refer to it as the PIAAC. Figure 2 illustrates the image quality improvement brought by the PIAAC design, and also compares it to a classical apodization technique.

\subsection{The PIAAC hybrid design}

There is however a serious issue with the concept presented above: on the first mirror, the edge exhibits a very sharp "bend", necessary to spread the light which is in the very outer portion of the incoming beam into a much larger area in the exit pupil. This sharp feature brings several problems:

- Optics manufacturing. The "bend" is critical to the apodization but extremely difficult to polish

- Alignement sensitivity. Since the mirror shape changes over small distances at this "bend", optics alignement tolerances become very strict.

- Chromaticity. This problem, unlike the others, goes beyond engineering considerations, and can fundamentally limit the PIAAC's performance. The "bend" is sharp enough to introduce diffractive (as opposed to geometrical) effects (Vanderbei 2005). 
Since these effects are chromatic, they would restrict the spectral bandwidth over which the required contrast level can be maintained.

The presence of this "bend" is due to the PIAA unit attempting to produce, in the output pupil, extremely dark areas (at the edge of the apodized pupil). A practical solution is therefore to share the apodization between a PIAA unit and classical apodizer mask. In this scheme, refered to as the "hybrid PIAAC", the PIAA unit does most of the apodization, but leaves some light in the outer parts of the beam (typically $1 \%$ of the surface brightness at the center of the beam). This makes the bend much smoother and also spreads it over a wider area. A classical apodizer can then "touch up" the outer parts of the beam to obtain the desired amplitude profile.

Since the classical apodizer mainly works in parts of the PIAA-apodized beam that are already faint, only a few percent (typically 5\%) of the light is lost. The performance loss is therefore minimal, while the practical gains are significant.

\section{Performance}

The PIAAC combines many desirable advantages:

- Small IWA. Efficient detection is possible as close as $1.5 \lambda / d$ for a $10^{10}$ contrast.

- Full throughput. As explained above, the only losses are due to the "touch up" classical apodizer (typically 5\%) and unavoidable reflections on mirrors.

- Achromaticity. The apodization relies on geometrics optics, and is therefore achromatic. We note that this is not exactly true since diffraction propagations effects do happen. However, we have verified that, with a good hybrid PIAAC design, chromatic effects are well below the $10^{-10}$ PSF contrast level over large (20\% or more) spectral bandwidths. Of course, other sources of chromaticity typically exist in a full system (telescope optics), and might restrict the useful bandwidth to less than $10 \%$.

- 360 degree search area. All of the field is used at once.

- Low sensitivity to low order aberrations. This is especially important since stars are partially resolved. If uncorrected telescope pointing error are especially large, the PIAAC's focal plane occulter diameter can be increased.
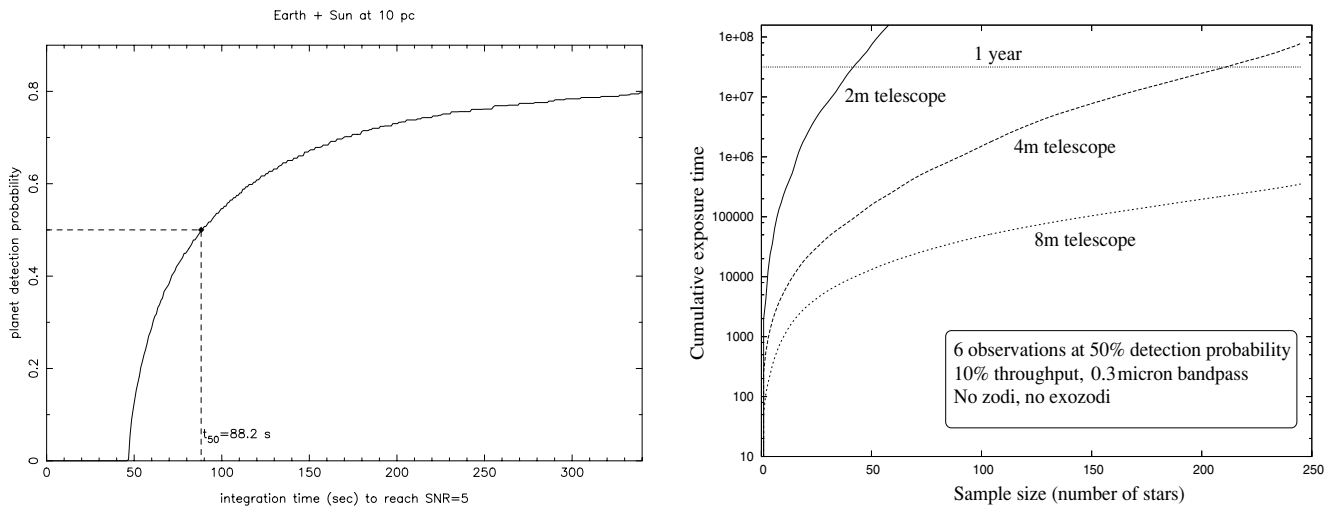

Figure 3. Left: Evolution of the planet detection probability (with a signal to noise ratio $S N R=5)$ as a function of exposure time for an Earth-Sun system at 10pc $\left(m_{V}=5,1 \mathrm{mas}\right.$ angular diameter, 100 mas maximum angular separation) observed by a $4 \mathrm{~m}$ telescope. The bandwidth is $\Delta \lambda=0.3 \mu \mathrm{m}$ and the central wavelength is $\lambda=0.5 \mu \mathrm{m}$. Right: Cumulative exposure time required for the survey of a sample of up to 250 stars for ETPs. 


\subsection{Direct imaging survey of ETPs with a PIAAC}

We summarize here results described in a recent paper by our team: Martinache et al. (2005).

We first consider the observation of a Sun-Earth system at $10 \mathrm{pc}$. We assume that the planet position along its orbit is unknown: the exposure time necessary for detection (defined by $\mathrm{SNR}=5$ on the image) cannot be computed. Instead, we can compute the planet detection probability as a function of integration time. Results for a PIAAC system on a "perfect" $4 \mathrm{~m}$ space telescope are shown in figure 3 (left). The combination of small IWA, high throughput, 360 degree search area results in a $50 \%$ detection probability in just over one minute of integration time.

Likewise, the integration time necessary to reach a $50 \%$ detection probability can be computed for each star of a target list. As shown in figure 3 (right), using somewhat realistic assumption ( 6 individual observations, each at the $50 \%$ detection probability, required per target; $10 \%$ system throughput; 1 year total integration time available), we find that Earth-like planets could be imaged around about 40 stars with a $2 \mathrm{~m}$ telescope, and around more than 200 stars with a $4 \mathrm{~m}$ telescope.

\subsection{Simulated images of a nearby system}

In this section, simulated images for the observation of HIP4569, a Sun-like star at 8.7pc, are shown. An Earth-like planet was artificially placed 1.04 AU from the star (distance where the stellar flux recieved by the planet equals the solar flux received by the Earth). The simulated images include the following assumptions:

- Stellar diameter was taken into account (slightly increases coronagraphic leaks).

- An exo-zodiacal cloud similar to our zodiacal cloud was placed around the star.

- Photon noise was included

- Telescope+camera throughput $=0.25$

- $0.2 \mu \mathrm{m}$ bandpass centered at $0.55 \mu \mathrm{m}$.

- 2 hr exposure time per image.

The images shown in figure 4 illustrate the ability of a small size telescope to directly detect Earth-like planets with a PIAAC. Visual inspection of the images shows that the detection of HIP4569's hypothetical companion requires either a PIAAC on a $2 \mathrm{~m}$ telescope or a classical apodizer on a $6 \mathrm{~m}$ telescope. This factor of 3 in telescope size is due to the lower throughput of the classical apodizer (10\% vs. $90 \%$ : a PIAAC on a $2 \mathrm{~m}$ telescope collects as many photons as a classical apodizer of a $6 \mathrm{~m}$ telescope) and larger inner working angle $(4 \lambda / d$ vs. $1.5 \lambda / d)$.

As the telescope size increases, the central star becomes partially resolved: on an $8 \mathrm{~m}$ diameter telescope, the stellar radius is $4 \%$ of $\lambda / d$. The coronagraphic leaks then increase for the PIAAC, and concentric diffraction rings appear in the image. To mitigate this problem, the diameter of the focal plane occulter in the PIAAC may be slightly increased (which slightly increases the IWA). These feature are however fainter than the exoziacal background: even in the $8 \mathrm{~m}$ telescope case without oversizing of the focal plane occulter, the integrated residual starlight is 6 times fainter than the integrated residual exozodiacal light. It is therefore unlikely that stellar angular size would limit the detection efficiency.

Figure 4 also shows that the PIAAC's improved angular resolution allows a better separation between the planet's light and the exozodiacal light. This benefit is especially important for systems with high zodi content, or inclined orientations. Another advantage of any high throughput coronagraph is to allow efficient sensing of the unwanted speckles in the focal plane. In an instrument aimed at achieving $10^{10}$ PSF contrast, it is critical to quickly sense speckles at this level, so that a command can be issued to the system's 


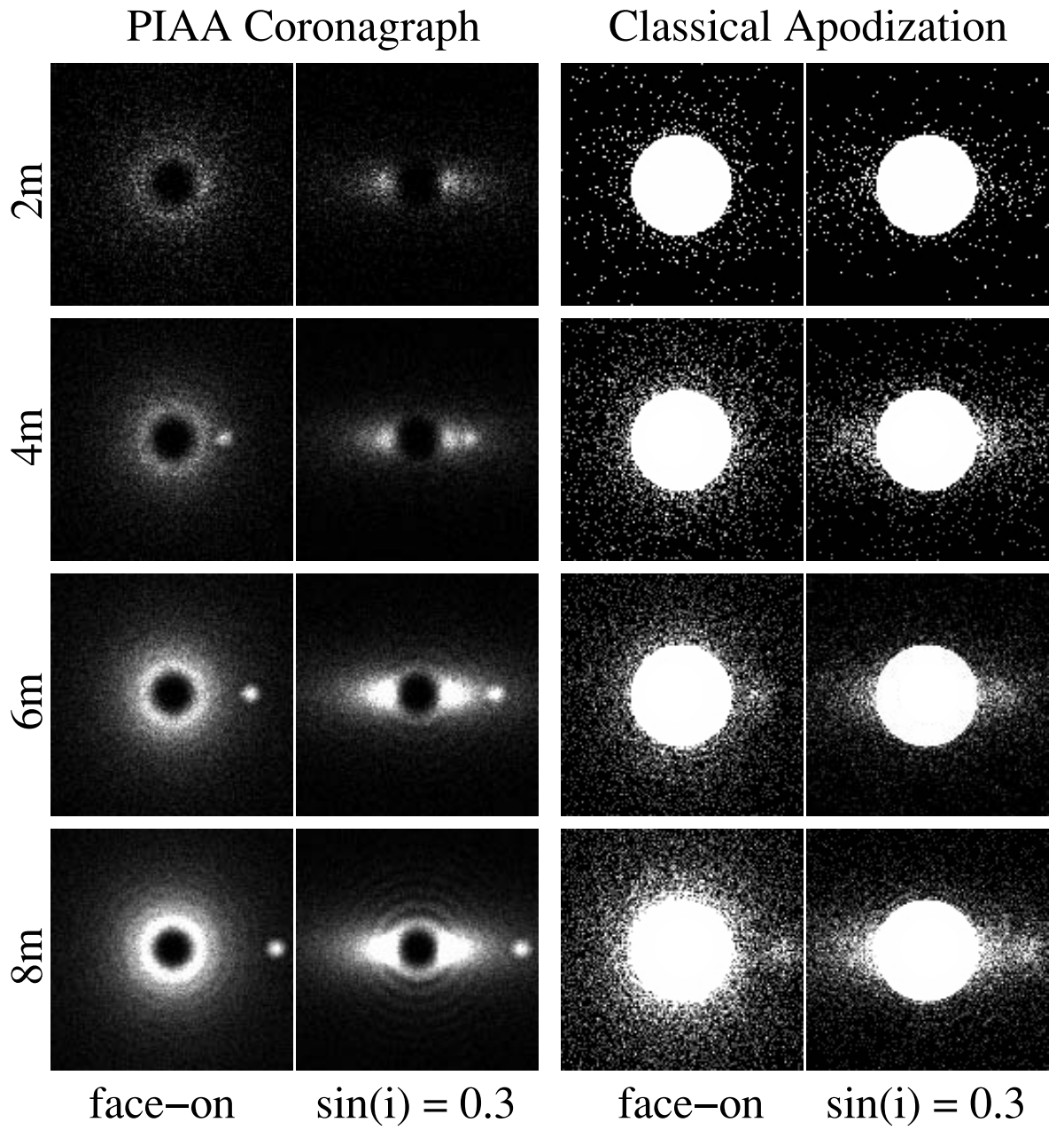

Figure 4. Simulated $0.55 \mu \mathrm{m}$ images of HIP4569 and an hypothetical Earth-type planet at maximum elongation for telescope sizes ranging from $2 \mathrm{~m}$ to $8 \mathrm{~m}$. For each coronagraph/telescope size combination, images were produced in the optimistic face-on case (left) and the inclined $\sin (\mathrm{i})=0.3$ case (right). See text for details.

deformable mirror to cancel this speckle. With the PIAAC's nearly-100\% throughput, the loop can be closed faster: the wavefront correction is more efficient.

\section{Conclusion}

We have shown in this paper that the PIAAC is theoretically highly efficient for direct imaging of ETPs from space (TPF-C mission). Its performance are close to what may be theoretically the "limit" of what a coronagraph can achieve: the off-axis PSF is diffraction-limited $(\lambda / d$ width) at nearly-100\% throughput very close to the optical axis (from $\approx 1.5 \lambda / d)$.

Although the design shown in this paper is targeted at observation at $\approx 10^{10}$ contrast, the PIAAC is also a very good candidate for ground-based observation with efficient adaptive optics systems, where the PSF contrast goal is more modest $\left(\approx 10^{6}\right.$ at most). In this case, the main advantages if the PIAAC is to allow efficient detection of faint 
compations at smaller distances than what would be possible with other coronagraphs. Its low sensitivity to tip-tilt errors is especially attractive since the performance of other small-IWA coronagraphs is often limited by uncorrected tip-tilt residuals.

\section{Acknowledgements}

This work was carried out under JPL contract numbers 1254445 and 1257767 for development of technologies for the Terrestrial Planet Finder Mission, with the support and hospitality of the National Astronomical Observatory of Japan.

\section{References}

Galicher, R., Guyon, O., Otsubo, M., Suto, H., \& Ridgway, S. 2005, PASP, 117, 411

Guyon, O., Pluzhnik, E.A., Galicher, R., Martinache, F., Ridgway, S.T., \& Woodruff, R.A. 2005, ApJ 622,744

Guyon, O. 2003, A\&SA 404, 379

Kasdin, N.J., Vanderbei, R.J., Spergel, D.N., \& Littman, M.G. 2003, ApJ 582, 1147

Martinache, F., Guyon, O., Pluzhnik, E.A., Galicher, R., \& Ridgway, S.T. 2005, Accepted for publication in ApJ

Traub, W.A. \& Vanderbei, R.J. 2003, ApJ 599, 695

Vanderbei, R.J. \& Traub, W.A. 2005, ApJ 626, 1079

Vanderbei, R.J. 2005, astro-ph/0506550, accepted for publication in ApJ

\section{Discussion}

: Why use an image image mask ?

GUYON: The image mask is needed to remove the starlight in the "intermediate" focal plane of the PIAAC. Without the focal plane mask, the inverse PIAA and direct PIAA would cancel each other, and an Airy disk (with its rings) would be left in the final focal plane.

: You seem more optimistic about the broadband performance than Roger Angel showed us in his talk.

GUYON: This is still work that is being done. I just showed you our latest designs, which are better than the ones we got a few weeks ago: we can now maintain a "null" better than $10^{-10}$ over a $20 \%$ bandwidth.

Ruslan Belikov: Is a Fresnel analysis accurate enough to produce all the diffraction problems you might encounter?

GuYON: The diffraction propagation we performed did not make the Fresnel approximation, so it should be quite accurate. Even if we've missed something, it should be small (much less $\lambda / 1000$ ), and well within the range of a wavefront control system.

Claude Aime: In your coronagraph performance comparative slide, you showed the Roddier\&Roddier phase mask has high throughput and small IWA, but it doesn't work for a large bandwidth

GuYOn: Yes, I did not mention about chromaticity in coronagraphs. The Roddier\&Roddier actually has another issue which makes it unsuitable for direct imaging of ETPs: it is very sensitive to tip-tilt. The angular diameter of a star is sufficient to prevent high contrast in the Roddier\&Roddier. 

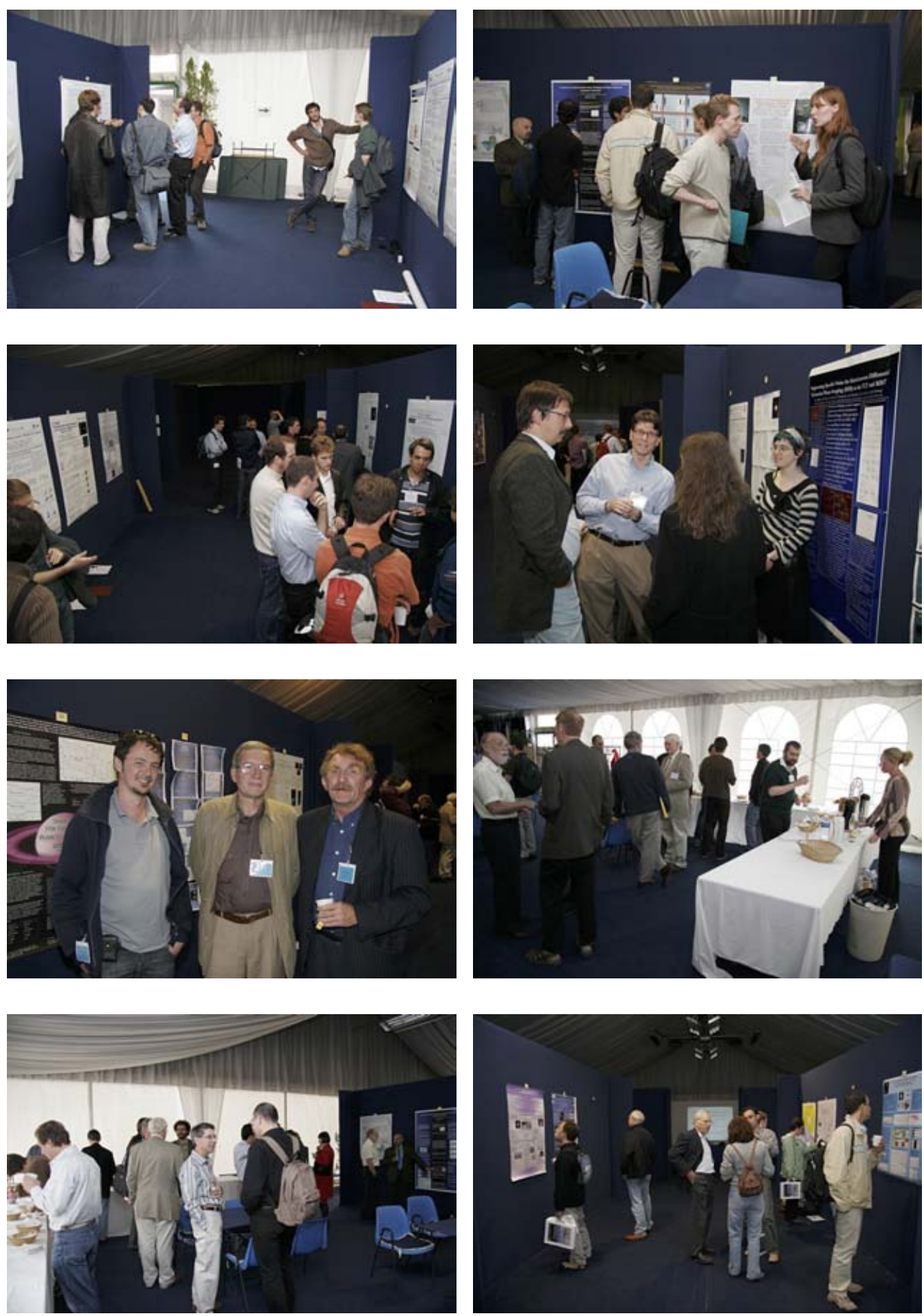

All photographs: Laurent Thareau [1.thareau@free.fr]. 